\title{
Prevalência e fatores associados à hipertensão arterial em idosos de municípios da Amazônia Legal, MT
}

\author{
Prevalence and factors associated with hypertension in the elderly from municipalities in the Legal \\ Amazon region, MT, Brazil
}

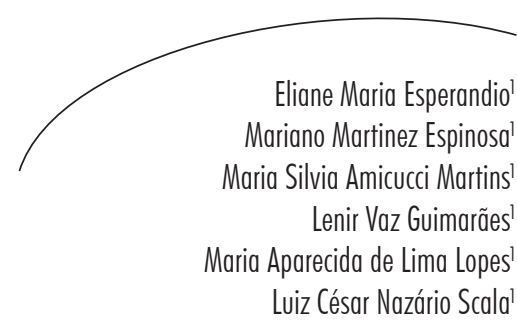

Resumo

Este estudo teve como objetivo analisar as características epidemiológicas da hipertensão arterial e os fatores associados na população idosa residente nos municípios da Amazônia Legal no Estado de Mato Grosso - Alta Floresta, Sinop, Sorriso e Diamantino. Trata-se de estudo transversal, de base populacional, realizado por meio de inquérito domiciliar com 312 idosos. A amostra da pesquisa foi obtida por amostragem complexa. Consideraram-se hipertensos aqueles que apresentaram pressão arterial $\geq 140 / 90 \mathrm{mmHg}$ e/ou que referiram uso de medicação anti-hipertensiva. A análise de regressão de Poisson foi utilizada para testar a associação entre a hipertensão e as variáveis independentes. A prevalência da hipertensão foi de $67,4 \%$. As variáveis associadas à hipertensão nos homens foram situação conjugal $(p=0,012)$ e índice de massa corporal $(\mathrm{p}=0,025)$. Já entre as mulheres, foram faixa etária $(\mathrm{p}=0,026)$, tabagismo $(\mathrm{p}=0,030)$ e índice de massa corporal $(\mathrm{p}=0,034)$. Entre os idosos, observou-se que $78,3 \%$ tinham conhecimento de sua condição de hipertenso; destes, 97,7\% estavam em tratamento. Entre os que estavam em tratamento, apenas $43,7 \%$ apresentavam sua pressão arterial controlada. A alta prevalência da hipertensão, a associação a fatores de risco cardiovascular, assim como o baixo percentual de idosos com pressão arterial controlada, reforçam a necessidade de implementar políticas públicas, visando a ações de prevenção, controle e promoção da saúde da população idosa.

Abstract

This paper aimed to analyze the epidemiological characteristics of hypertension and associated factors in an elderly population living in municipalities of the Legal Amazon region in Mato Grosso state, Brazil - Alta Floresta, Sinop, Sorriso and Diamantino. Cross-sectional, population-based study, conducted through a household survey with

Palavras-chave:

Epidemiologia. Hipertensão. Fatores de Risco. Idoso.

\footnotetext{
Programa de Pós-graduação em Saúde Coletiva, Instituto de Saúde Coletiva. Universidade Federal de Mato Grosso. Cuiabá, MT, Brasil.

Este artigo é parte da dissertação do mestrado de Eliane Maria Esperandio pelo Programa de Pósgraduação em Saúde Coletiva da Universidade Federal de Mato Grosso.
}

Correspondência / Correspondence

Universidade Federal de Mato Grosso

Av. Fernando Correa da Costa $\mathrm{s} / \mathrm{n}^{\circ}$, Coxipó da Ponte

78060-900 Cuiabá, MT, Brasil

E-mail: elianesperandio@hotmail.com 
312 elderly. The survey sample was obtained by simple sampling and cluster in stages. The individuals who had $\geq 140 / 90 \mathrm{mmHg}$ and / or reported use of antihypertensive medication were considered hypertensive. Poisson regression analysis was used to test the association between hypertension and the independent variables. The prevalence of hypertension was $67.4 \%$. The variables were associated with hypertension in males were marital status $(p=0.012)$ and body mass index $(p=0.025)$. Among females, were age $(p=0.026)$, smoking $(p=0.030)$ and body mass index $(p=0.034)$. Among the elderly, $78.3 \%$ were aware of their condition, and among these, $97.9 \%$ underwent treatment. But among those who were treated only $43.7 \%$ had their blood pressure controlled. The high prevalence of hypertension and its association with cardiovascular risk factors, as well as the percentage of elderly with controlled blood pressure, reinforce the need for implementation of public policies aimed at prevention, control and promotion of health in the elderly population.

\section{INTRODUÇÃO}

O envelhecimento populacional é uma importante mudança demográfica observada na atualidade. Existem cerca de 600 milhões de idosos vivendo no mundo e, destes, 370 milhões estão em países em desenvolvimento. ${ }^{1} \mathrm{O}$ processo de transição demográfica influencia a transição epidemiológica e a carga de doenças, em especial no idoso. Em 2007, cerca de 70\% das mortes no Brasil foram atribuídas às doenças crônicas não transmissíveis (DCNT), especialmente as doenças cardiovasculares, uma das causas mais frequentes de mortalidade entre os idosos. ${ }^{2}$

A hipertensão arterial sistêmica (HAS) é um importante fator de risco cardiovascular modificável. Devido a sua alta prevalência e sua relação causal com todas as doenças cardiovasculares, a hipertensão arterial é o principal fator de risco para a mortalidade em todo o mundo, revelando-se como importante problema de saúde pública. ${ }^{3}$

Embora seja apontado que doenças e limitações não são resultados inevitáveis do envelhecimento, ${ }^{4}$ existem amplas evidências de quealterações próprias do envelhecimento tornam o indivíduo mais propenso ao desenvolvimento de HAS, sendo esta a principal doença crônica nessa população. Estima-se que no Brasil mais
Key words: Epidemiology. Hypertension. Risk Factors. Elderly. de $60 \%$ dos idosos sejam hipertensos. ${ }^{5}$ Nesse contexto, a HAS, além de ser um dos principais fatores de risco para a mortalidade nessa faixa etária, também causa prejuízo à qualidade de vida, fato que muitas vezes retira o valor da longevidade conquistada. ${ }^{6}$

Resultados de diversos ensaios clínicos têm demonstrado que a intervenção terapêutica antihipertensiva é eficaz em diminuir a morbidade e a mortalidade cardiovascular. ${ }^{5}$ Apesar das evidências, estima-se que apenas um terço da população hipertensa tenha sua pressão arterial (PA) controlada, tanto em países desenvolvidos como nos em desenvolvimento. ${ }^{7}$

Vários trabalhos têm sido publicados na literatura nacional e internacional sobre a epidemiologia da HAS; 5,7 entretanto, comportamentos de saúde em idosos hipertensos têm recebido pouca atenção. ${ }^{6}$ Conhecer a situação de saúde da população idosa é uma importante estratégia para melhorar a qualidade de saúde da pessoa idosa e poderá ser útil no planejamento de políticas de prevenção e controle da hipertensão.

O objetivo deste estudo foi analisar as características epidemiológicas da hipertensão arterial sistêmica (HAS) e os fatores associados na população idosa residente em municípios da Amazônia Legal, Estado de Mato Grosso. 


\section{MÉTODOS}

Trata-se de estudo de delineamento transversal, de base populacional, realizado por meio de inquérito domiciliar, com 312 idosos de idade igual ou maior que 60 anos, de ambos os sexos, residentes na área urbana dos municípios de Alta Floresta, Sinop, Sorriso e Diamantino, localizados na região da Amazônia Legal no Estado de Mato Grosso, Brasil. O estudo compõe o projeto "Segurança alimentar e nutricional da população residente na área de influência da BR-163", realizado por pesquisadores da Universidade Federal de Mato Grosso em parceria com a Secretaria de Estado de Saúde de Mato Grosso e Secretarias de Saúde dos municípios estudados.

Os municípios selecionados situam-se na área de influência da rodovia BR-163, que liga a cidade de Cuiabá, capital do Estado de Mato Grosso, à cidade de Santarém, no Estado do Pará, importante área da Amazônia brasileira, tanto do ponto de vista econômico quanto ecológico. ${ }^{8}$

O tipo de amostragem probabilística adotado pelo planejamento amostral do projeto matriz foi o método conglomerado em dois estágios. Trata-se de estudo com delineamento amostral complexo, em que as unidades primárias de amostragem foram os setores censitários e as unidades secundárias, os domicílios.

O tamanho da amostra em cada município foi obtido considerando o nível de confiança de $95 \%$ e uma proporção de $50 \%$, com um erro de estimação de 3,5\%. Para cada município, foi determinado o número de domićlios a serem sorteados na pesquisa, com acréscimo de $20 \%$ para possíveis perdas na etapa da coleta de dados. Em seguida, utilizou-se um cadastro atualizado, por meio de visita in loco, para certificar a existência e ocupação dos setores e respectivos domicílios, procedendo-se ao sorteio aleatório dos mesmos.
A coleta dos dados foi realizada por uma dupla de entrevistadores devidamente treinada. Os dados foram coletados nos domicílios por meio de entrevista, com aplicação de formulários previamente testados, no período de fevereiro a outubro de 2007. Foram obtidas informações sobre identificação do indivíduo, variáveis demográficas, socioeconômicas, estilo de vida, morbidade referida e medidas antropométricas e de pressão arterial. As variáveis demográficas e socioeconômicas estudadas foram: sexo, idade, raça/cor, situação conjugal, escolaridade e renda familiar mensal per capita em salários mínimos.

As categorias para o tabagismo foram: fumantes, não fumantes e ex-fumantes. ${ }^{9} \mathrm{O}$ consumo de bebidas alcoólicas foi categorizado em "sim" e "não", questionado em relação aos 30 dias anteriores à entrevista. A atividade física no lazer foi categorizada em "sedentário" e "não sedentário", considerado sedentário o indivíduo que referiu não praticar qualquer esporte ou exercício físico pelo menos uma vez na semana. ${ }^{10}$

O peso foi obtido por meio de balança eletrônica marca Tanita, modelo UM 080, com capacidade para $150 \mathrm{Kg}$ e variação de $0,1 \mathrm{Kg}$; para a estatura, o instrumento utilizado foi o estadiômentro portátil Seca. O peso e a estatura foram obtidos com indivíduos usando roupas leves, descalços, em posição ortostática. ${ }^{11}$ A circunferência da cintura foi medida segundo técnica de Callaway, ${ }^{12}$ utilizando fita antropométrica marca Mabbis, modelo Gulick. Para a análise, calculou-se o índice de massa corporal $\left(\mathrm{IMC}=\mathrm{peso} / \mathrm{estatura}^{2}\right),{ }^{13}$ categorizando os indivíduos em dois grupos: $<30 \mathrm{Kg} / \mathrm{m}^{2}$ e $\geq 30 \mathrm{Kg} / \mathrm{m}^{2}$. A circunferência da cintura foi considerada normal $(<80 \mathrm{~cm}$ e $<94 \mathrm{~cm})$, aumentada ( 80 a $88 \mathrm{~cm}$ e 94 a $102 \mathrm{~cm})$ e muito aumentada $(>88 \mathrm{~cm}$ e $>102 \mathrm{~cm})$ para mulheres e homens, respectivamente. ${ }^{13}$

Em relação ao conhecimento da condição de hipertensão, foram considerados os indivíduos 
que autorreferiram saber desta condição. Quanto ao tratamento, foram considerados os idosos que referiram uso de anti-hipertensivos e, em relação ao controle da PA, foram considerados aqueles que apresentaram $\mathrm{PA}<140 / 90 \mathrm{mmHg}$ na aferição.

A PA foi aferida três vezes com intervalo mínimo de três minutos entre cada uma, utilizando o aparelho semiautomático de marca OMRON-HEM 711AC, considerando-se para análise a média das duas últimas medidas. Foram considerados hipertensos os indivíduos que apresentaram PA sistólica $\geq 140 \mathrm{mmHg}$ e/ou PA diastólica $\geq 90 \mathrm{mmHg}$ e indivíduos que referiram uso regular de medicação anti-hipertensiva. ${ }^{14}$

Os dados foram digitados em duplicata, no pacote estatístico Epi Info 2000, que permite a análise da consistência dos dados. Todas as análises estatísticas foram realizadas no programa Stata versão 10.0, utilizando a ponderação da amostra e considerando o efeito do desenho amostral segundo os procedimentos para amostras complexas (svy), o qual permitiu incorporar os pesos distintos das observações. ${ }^{15}$

Para verificar a existência de associação entre a variável dependente HAS e demais variáveis independentes do estudo, foram estimadas as razões de prevalências com respectivos intervalos de confiança de $95 \%$ por meio da regressão de Poisson, com nível de significância de $\mathrm{p}<0,05 .{ }^{15}$ As variáveis que apresentaram $\mathrm{p}<0,20$ foram incluídas em um modelo de regressão múltipla, utilizando o método de adição progressiva das variáveis (stepwise forward), tendo como critério para permanência no modelo de regressão as variáveis que apresentassem nível de significância menor que $5 \%$. Para analisar o nível de conhecimento, tratamento e controle da pressão arterial, foram calculadas as proporções.

Opresenteestudo foi financiadopelo Conselho Nacional de Desenvolvimento Científico e Tecnológico (CNPq) e aprovado pelo Comitê de Ética e Pesquisa do Hospital Universitário Júlio
Muller (UFMT), sob protocolo $\mathrm{n}^{\circ}$ 230/CEPHUJM/06. Todos os participantes assinaram o Termo de Consentimento Livre e Esclarecido.

\section{RESULTADOS}

Dos 312 indivíduos elegíveis para o estudo, participaram 273 (87,5\%), havendo uma perda de 39 idosos por motivo de recusa ou ausência no momento da visita ao domicílio. A média de idade dos participantes foi de $67,7 \pm 6,4$ anos, sendo $68,6 \pm 6,8$ anos para homens e $66,9 \pm 5,9$ anos para mulheres. A população estudada foi constituída de 55,3\% de mulheres. Entre os entrevistados, $64,8 \%$ estavam na faixa etária de 60 a 69 anos e 64,8\% eram casados. Com relação à variável raça/cor, 57,5\% autorreferiram como sendo pardos e negros e $42,5 \%$ como brancos. Observou-se que $54,9 \%$ dos indivíduos tiveram de um a quatro anos de estudo, enquanto 31,5\% tiveram menos de um ano de estudo. Entre os indivíduos entrevistados 34,1\% tinham renda familiar mensal per capita superior a 1,2 salários mínimos.

A prevalência da HAS foi de $67,4 \%$ (IC 95\%: $61,8-73,0)$, sendo para o sexo masculino $68,0 \%$ (IC 95\%: 57,9-78,2) e para o feminino, 66,9\% (IC 95\%: 57,7-76,1). Quando analisada a prevalência da HAS entre os idosos do sexo masculino, observou-se que a situação conjugal, o índice de massa corporal (IMC) e a circunferência da cintura associaram-se positivamente com a hipertensão $(\mathrm{p}<0,05)$. Maior prevalência da hipertensão foi observada entre os idosos não casados, quando comparada aos casados $(\mathrm{p}<0,036)$. Entre os idosos que apresentaram IMC $\geq 30 \mathrm{Kg} / \mathrm{m}^{2}$, a prevalência da hipertensão foi $56 \%$ maior em relação aos que apresentaram $\mathrm{IMC}<30 \mathrm{Kg} / \mathrm{m}^{2}$. Da mesma forma, verificouse que a prevalência de hipertensão nos idosos com circunferência da cintura aumentada foi $39 \%$ maior, se comparada aos que apresentaram circunferência da cintura normal (tabela 1). 
Tabela 1 - Prevalência de hipertensão arterial, segundo variáveis demográficas, socioeconômicas, de estilo de vida e antropométricas em idosos do sexo masculino residentes nos municípios de Alta Floresta, Sinop, Sorriso e Diamantino-MT, 2007.

\begin{tabular}{|c|c|c|c|c|c|c|}
\hline \multirow{2}{*}{ Variável } & \multirow{2}{*}{$\mathrm{n}$} & \multicolumn{2}{|c|}{ Hipertensão } & \multirow{2}{*}{$\mathrm{RP}$} & \multirow{2}{*}{ IC } & \multirow{2}{*}{ p-valor } \\
\hline & & $\mathrm{n}$ & $\%$ & & & \\
\hline \multicolumn{7}{|l|}{ Faixa etária } \\
\hline 60-69 anos & 75 & 50 & 66,7 & 1,00 & & \\
\hline 70 anos e mais & 47 & 33 & 70,2 & 1,05 & $0,79-1,41$ & 0,688 \\
\hline \multicolumn{7}{|l|}{ Raça/cor } \\
\hline Branco & 53 & 30 & 56,6 & 1,00 & & \\
\hline Não branco & 69 & 53 & 76,8 & 1,36 & $0,91-2,03$ & 0,115 \\
\hline \multicolumn{7}{|c|}{ Escolaridade (ano de estudo) } \\
\hline$<1$ & 40 & 28 & 70,0 & 1,00 & & \\
\hline 1 a 4 & 68 & 46 & 67,6 & 0,97 & $0,67-1,39$ & 0,831 \\
\hline$>4$ & 14 & 9 & 64,3 & 0,92 & $0,55-1,52$ & 0,705 \\
\hline \multicolumn{7}{|l|}{ Renda familiar per capita } \\
\hline$<0,8 \mathrm{SM}$ & 42 & 27 & 64,3 & 1,00 & & \\
\hline 0,8 a $1,2 \mathrm{SM}$ & 42 & 29 & 69,0 & 1.07 & $0.81-1.42$ & 0.565 \\
\hline$>1,2 \mathrm{SM}$ & 38 & 27 & 71,1 & 1.10 & $0.77-1.58$ & 0.529 \\
\hline \multicolumn{7}{|l|}{ Situação conjugal } \\
\hline Casado & 96 & 61 & 63,5 & 1,00 & & \\
\hline Não casado & 26 & 22 & 84,6 & 1,33 & $1,02-1,73$ & 0,036 \\
\hline \multicolumn{7}{|l|}{ Tabagismo } \\
\hline Não fumante & 29 & 20 & 69,0 & 1,00 & & \\
\hline Fumante & 35 & 23 & 65,7 & 0,95 & $0,69-1,31$ & 0,729 \\
\hline Ex-fumante & 58 & 40 & 69,0 & 1,00 & $0,70-1,42$ & 1,000 \\
\hline \multicolumn{7}{|c|}{ Consumo de bebida alcoólica } \\
\hline Não & 80 & 59 & 73,8 & 1,00 & & \\
\hline $\operatorname{Sim}$ & 42 & 24 & 57,1 & 0,77 & $0,48-1,26$ & 0,251 \\
\hline \multicolumn{7}{|c|}{ Atividade física no lazer } \\
\hline Não sedentário & 64 & 42 & 65,6 & 1,00 & & \\
\hline Sedentário & 58 & 41 & 70,7 & 1,08 & $0,78-1,48$ & 0,601 \\
\hline \multicolumn{7}{|c|}{ Índice de massa corporal } \\
\hline$<30 \mathrm{Kg} / \mathrm{m}^{2}$ & 109 & 70 & 64,2 & 1,00 & & \\
\hline$\geq 30 \mathrm{Kg} / \mathrm{m}^{2}$ & 13 & 13 & 100,0 & 1,56 & $1,26-1,92$ & 0,002 \\
\hline \multicolumn{7}{|c|}{ Circunferência da cintura } \\
\hline Normal & 72 & 44 & 61,1 & 1,00 & & \\
\hline Aumentada & 30 & 22 & 73,3 & 1,20 & $1,03-1,39$ & 0,024 \\
\hline Muito aumentada & 20 & 17 & 85,0 & 1,39 & $0,97-1,99$ & 0,067 \\
\hline
\end{tabular}

RP: razão de prevalência; IC: intervalo com 95\% de confiança; SM: salário mínimo no momento da pesquisa (2007) no município de Sinop, correspondente a $\mathrm{R} \$ 350,00$; demais municípios (Alta Floresta, Sorriso e Diamantino), correspondente a R $\$ 380,00$. 
Quanto à análise da prevalência da HAS entre mulheres idosas (tabela 2), verificou-se aumento da prevalência de hipertensão com o aumento da idade $(p=0,017)$. Observou-se que as idosas classificadas como ex-fumantes apresentaram maior prevalência de hipertensão em relação às não fumantes ( $\mathrm{RP}=1,2$; IC:1,02-1,39). Entre as idosas que apresentaram IMC $\geq 30 \mathrm{Kg} / \mathrm{m}^{2}$, a prevalência de hipertensão foi $69 \%$ maior em relação às que apresentaram IMC $<30 \mathrm{Kg} / \mathrm{m}^{2}$. Verificouse que a prevalência de hipertensão nas idosas com circunferência da cintura muito aumentada foi $85 \%$ maior, quando comparadas àquelas que apresentaram circunferência da cintura normal. 
Tabela 2 - Prevalência de hipertensão arterial, segundo variáveis demográficas, socioeconômicas, de estilo de vida e antropométricas em idosos do sexo feminino residentes nos municípios de Alta Floresta, Sinop, Sorriso e Diamantino-MT, 2007.

\begin{tabular}{|c|c|c|c|c|c|c|}
\hline \multirow{2}{*}{ Variável } & \multirow{2}{*}{$\mathrm{n}$} & \multicolumn{2}{|c|}{ Hipertensão } & \multirow{2}{*}{$\mathrm{RP}$} & \multirow{2}{*}{ IC } & \multirow{2}{*}{ p-valor } \\
\hline & & $\mathrm{n}$ & $\%$ & & & \\
\hline \multicolumn{7}{|l|}{ Faixa etária } \\
\hline 60-69 anos & 102 & 63 & 61,8 & 1,00 & & \\
\hline 70 anos e mais & 49 & 38 & 77,6 & 1,25 & $1,06-1,49$ & 0,017 \\
\hline \multicolumn{7}{|l|}{ Raça/cor } \\
\hline Branco & 63 & 42 & 66,7 & 1,00 & & \\
\hline Não branco & 88 & 59 & 67,0 & 1,01 & $0,80-1,26$ & 0,954 \\
\hline \multicolumn{7}{|c|}{ Escolaridade (ano de estudo) } \\
\hline$<1$ & 46 & 29 & 63,0 & 1,00 & & \\
\hline 1 a 4 & 82 & 56 & 68,3 & 1,08 & $0,90-1,31$ & 0,349 \\
\hline$>4$ & 23 & 16 & 69,6 & 1,10 & $0,76-1,61$ & 0,557 \\
\hline \multicolumn{7}{|c|}{ Renda familiar per capita } \\
\hline$<0,8 \mathrm{SM}$ & 46 & 30 & 65,2 & 1,00 & & \\
\hline 0,8 a $1,2 \mathrm{SM}$ & 50 & 28 & 56,0 & 0.86 & $0.59-1.25$ & 0.374 \\
\hline$>1,2 \mathrm{SM}$ & 55 & 43 & 78,2 & 1.20 & $0.91-1.57$ & 0.157 \\
\hline \multicolumn{7}{|l|}{ Situação conjugal } \\
\hline Casado & 81 & 49 & 60,5 & 1,00 & & \\
\hline Não casado & 70 & 52 & 74,3 & 1,23 & $0,97-1,56$ & 0,080 \\
\hline \multicolumn{7}{|l|}{ Tabagismo } \\
\hline Não fumante & 101 & 66 & 65,3 & 1,00 & & \\
\hline Fumante & 18 & 10 & 55,6 & 0,85 & $0,50-1,44$ & 0,491 \\
\hline Ex-fumante & 32 & 25 & 78,1 & 1,19 & $1,02-1,39$ & 0,027 \\
\hline \multicolumn{7}{|c|}{ Consumo de bebida alcoólica } \\
\hline Não & 134 & 92 & 68,7 & 1,00 & & \\
\hline $\operatorname{Sim}$ & 17 & 9 & 52,9 & 0,77 & $0,48-1,24$ & 0,236 \\
\hline \multicolumn{7}{|c|}{ Atividade física no lazer } \\
\hline Não sedentário & 134 & 42 & 62,7 & 1,00 & & \\
\hline Sedentário & 17 & 53 & 70,2 & 1.12 & $0.85-1.48$ & 0.367 \\
\hline \multicolumn{7}{|c|}{ Índice de massa corporal } \\
\hline$<30 \mathrm{Kg} / \mathrm{m}^{2}$ & 115 & 66 & 57,4 & 1,00 & & \\
\hline$\geq 30 \mathrm{Kg} / \mathrm{m}^{2}$ & 36 & 35 & 97,2 & 1,69 & $1,35-2,12$ & 0,001 \\
\hline \multicolumn{7}{|c|}{ Circunferência da cintura } \\
\hline Normal & 42 & 19 & 45,2 & 1,00 & & \\
\hline Aumentada & 42 & 26 & 61,9 & 1,37 & $0,81-2,30$ & 0,196 \\
\hline Muito aumentada & 67 & 56 & 83,6 & 1,85 & $1,17-2,92$ & 0,016 \\
\hline
\end{tabular}

RP: razão de prevalência; IC: intervalo com 95\% de confiança; SM: salário mínimo no momento da pesquisa (2007) no município de Sinop, correspondente a $\mathrm{R} \$ 350,00$; demais municípios (Alta Floresta, Sorriso e Diamantino), correspondente a R $\$ 380,00$. 
A tabela 3 apresenta os fatores associados ao desfecho após análise no modelo de regressão de Poisson. No sexo masculino, as categorias que se mantiveram significantes na associação com a HAS foram: os não casados $(\mathrm{p}=0,012) \mathrm{e}$ os que apresentaram IMC $<30 \mathrm{Kg} / \mathrm{m}^{2}(\mathrm{p}=0,025)$. No sexo feminino, as variáveis associadas à HAS foram: ter 70 anos e mais de idade $(p=0,026)$, ser ex-fumante $(\mathrm{p}=0,030)$ e as que apresentaram $\mathrm{IMC}<30 \mathrm{Kg} / \mathrm{m}^{2}(\mathrm{p}=0,034)$.

Tabela 3 - Prevalência de hipertensão arterial ajustada por variáveis demográficas, socioeconômicas, de estilo de vida e antropométricas em idosos residentes nos municípios de Alta Floresta, Sinop, Sorriso e Diamantino-MT, 2007.

\begin{tabular}{lccc}
\hline Variável & RP & IC & p-valor \\
\cline { 2 - 2 } & ajustada & & \\
\hline
\end{tabular}

Masculino

Faixa etária*
60-69 anos
1,00
70 anos e mais
1,04
$0,79-1,38$
0,71

Situação conjugal

Casado

1,00

Não casado

1,32

$1,08-1,59$

0,012

Índice de massa corporal
$<30 \mathrm{Kg} / \mathrm{m}^{2}$
1,00
$\geq 30 \mathrm{Kg} / \mathrm{m}^{2}$
1,59

$1,07-2,34$

0,025

Feminino

Faixa etária
60-69 anos
1,00
70 anos e mais
1,28

$1,04-1,61$

0,026

Tabagismo

Não fumante

1,00

Fumante

$0,49-1,47$

0,504

Ex-fumante

1,20

$1,02-1,40$

0,030

Índice de massa corporal
$<30 \mathrm{Kg} / \mathrm{m}^{2}$
1,00
$\geq 30 \mathrm{Kg} / \mathrm{m}^{2}$
1,44

$1,03-2,00$

0,034

RP: razão de prevalência ajustada por todas as outras variáveis apresentadas nas tabelas 1 e 2; IC: intervalo com $95 \%$ de confiança; *variável de ajuste. 
$\mathrm{Na}$ tabela 4, observou-se que entre os 184 idosos hipertensos, 144 (78,3\%) tinham conhecimento de sua condição; 141 (97,9\%) estavam em tratamento; e 62 (43,7\%) apresentavam PA controlada. Quanto ao gênero, não houve diferenças estatísticas observadas quando considerados os idosos com conhecimento de sua condição, que faziam tratamento e apresentavam PA controlada. Em relação à escolaridade, os idosos com menos de um ano de estudo apresentaram menor controle da PA (30\%) do que os idosos com um ou mais anos de estudo (50\%), apresentando aumento estatisticamente significativo $(\mathrm{p}<0,05)$.

Tabela 4 - Prevalência, conhecimento, tratamento e controle da hipertensão arterial, segundo variáveis sexo e escolaridade em idosos residentes nos municípios de Alta Floresta, Sinop, Sorriso e Diamantino-MT, 2007.

\begin{tabular}{lccccccccc}
\hline \multirow{2}{*}{ Variável } & $\mathrm{n}$ & \multicolumn{2}{c}{ Prevalência } & \multicolumn{2}{c}{ Conhecimento } & \multicolumn{2}{c}{ Tratamento } & \multicolumn{2}{c}{ Controle } \\
\cline { 3 - 10 } & & $\mathrm{n}$ & $\%$ & $\mathrm{n}$ & $\%$ & $\mathrm{n}$ & $\%$ & $\mathrm{n}$ & $\%$ \\
\hline Total & 273 & 184 & 67,4 & 144 & 78,1 & 141 & 97,8 & 62 & 43,5 \\
Sexo & & & & & & & & & \\
Masculino & 122 & 83 & 68,0 & 59 & 71,0 & 58 & 98,0 & 25 & 43,0 \\
Feminino & 151 & 101 & 66,9 & 85 & 84,0 & 83 & 97,5 & 37 & 44,5 \\
Escolaridade (ano de estudo) & & & & & & & & \\
$<1$ & 86 & 57 & 66,1 & 46 & 80,5 & 43 & 93,3 & 13 & $30,0^{*}$ \\
$>1$ & 187 & 127 & 67,8 & 98 & 77,0 & 98 & 100,0 & 49 & 50,0 \\
\hline
\end{tabular}

$* \mathrm{p}<0,05$.

\section{DISCUSSÃO}

Neste estudo, observou-se alta prevalência de HAS (67,4\%) entre os idosos pesquisados. Destacam-se as taxas elevadas de prevalência de hipertensão em núcleos urbanos de pequeno porte, mas estes dados são semelhantes aos encontrados em outras cidades brasileiras de pequeno, ${ }^{16,17}$ médio $^{18}$ e grande porte ${ }^{19} \mathrm{e}$ em outros países para a mesma faixa etária ${ }^{20}$ e entre idosos com idade igual ou superior a 65 anos. ${ }^{21}$ Com o aumento da longevidade da população, ocorre uma agregação de outros fatores de risco, que culmina por comprometer a qualidade de vida dos idosos.

Estudo de Martins et al. ${ }^{22}$ em Sinop, município pesquisado neste estudo, observou prevalência de hipertensão de $23,2 \%$, apontando a necessidade de medidas de intervenção mais precoces na idade adulta, com o objetivo de modificar esse quadro desfavorável.

Em relação ao gênero, diferentemente do encontrado em outros estudos, ${ }^{23,24}$ observouse que a prevalência de HAS não apresentou diferença estatisticamente significativa, pois $68 \%$ dos homens e aproximadamente $67 \%$ das mulheres eram hipertensos. Brindela et al., ${ }^{25}$ na França, observaram maior prevalência de hipertensão entre os idosos do gênero masculino com idade $\geq 65$ anos.

$\mathrm{Na}$ análise de regressão múltipla, as variáveis que se associaram à HAS no gênero masculino foram: situação conjugal e IMC, enquanto que no feminino as variáveis faixa etária, tabagismo e IMC mantiveram associações significantes com a hipertensão arterial. 
A análise da situação conjugal no sexo masculino revelou que idosos não casados apresentaram prevalência de hipertensão $(84,6 \%)$ significativamente maior em relação aos casados $(63,5 \%)$. Para Dupre et al., ${ }^{26}$ a instabilidade conjugal diminui a renda, causa estresse, interfere no bem-estar emocional e no estilo de vida, precipitando doenças crônicas e mortalidade. Casamentos longos incentivam comportamentos saudáveis, reduzem o risco de doenças crônicas e promovem maior sobrevivência, particularmente entre os homens.

Alterações na composição corporal no idoso são um processo fisiológico que pode variar entre os indivíduos. As principais alterações referemse à massa muscular e ao padrão de distribuição de gordura corporal. ${ }^{27,28} \mathrm{~A}$ obesidade tem sido descrita como fator de risco que compromete a qualidade de vida dos idosos. Pessoas com excesso de peso têm maior probabilidade de desenvolver hipertensão arterial. ${ }^{29}$

No presente trabalho, observou-se uma associação positiva entre hipertensão arterial e obesidade em ambos os sexos $(p=0,025$ para homens e $\mathrm{p}=0,034$ para mulheres). De forma expressiva, observou-se que 100\% dos homens obesos e 97,2\% das mulheres obesas eram hipertensos. A relação entre obesidade e hipertensão tem sido relatada em diversos estudos. $^{24,30,31}$

Nas mulheres idosas, na análise por faixa etária, observou-se aumento da prevalência de HAS com o aumento da idade. Este achado é consistente com estudo realizado em países da América Latina e da Ásia entre idosos com 65 anos e mais e com pressão arterial medida. ${ }^{21}$ Nas idosas, a prevalência de hipertensão foi aproximadamente 1,3 vez maior na faixa etária igual ou superior a 70 anos, quando comparada à faixa etária de 6069 anos. Em estudo realizado na cidade de São Paulo $^{31}$ com idosas da mesma faixa etária e que autorreferiram hipertensão, observou-se que em mulheres com idade $\geq 75$ anos, a prevalência da hipertensão foi 1,3 vez maior quando comparada a mulheres com menos de 75 anos.

O envelhecimento propicia alterações estruturais e funcionais no sistema cardiovascular, predispondo ao desenvolvimento da HAS. Nas mulheres, a partir dos 45 anos, as alterações próprias do climatério conferem aumento da PA e do risco cardiovascular. ${ }^{32,33}$ Para Coylewright et al., ${ }^{33}$ a menopausa, associada ao aumento da rigidez arterial devido ao avanço da idade, pode ser responsável pela elevada prevalência de hipertensão em idosas.

Uma das limitações do presente estudo foi que o desenho de corte transversal não permitiu saber se os fatores associados à hipertensão antecederam a ocorrência da doença ou são, de alguma forma, consequentes à mesma. Esta observação é particularmente válida quanto à associação entre hipertensão arterial e tabagismo.

Neste estudo, verificou-se que as idosas exfumantes apresentaram maior prevalência de hipertensão em relação aos não fumantes e fumantes. Esse resultado pode ter ocorrido em consequência da causalidade reversa, pois as pessoas diagnosticadas com hipertensão arterial são orientadas a parar de fumar.

Em relação ao conhecimento, tratamento e controle da PA, no presente estudo observou-se que não houve diferença estatisticamente significante em relação ao gênero. Entre os homens hipertensos, verificou-se que $71 \%$ reconheciam essa condição e, destes, 98\% estavam em tratamento. Já entre as mulheres hipertensas, $84 \%$ sabiam ser hipertensas e, destas, 97,5\% estavam em tratamento. Entre os idosos em tratamento, observou-se controle da PA em $44,5 \%$ das mulheres, versus $43 \%$ dos homens, achado consistente com a literatura. ${ }^{25,34}$ 
A escolaridade desempenha importante papel nas condições de saúde, em decorrência de vários fatores como informações sobre promoção da saúde, prevenção das doenças, compreensão do problema e adesão ao tratamento. ${ }^{35}$ Neste estudo, quando observado o conhecimento dos idosos sobre a condição de hipertensos, e dos que os estavam tratando, não foram observadas diferenças estatisticamente significantes segundo a escolaridade. Já entre os hipertensos que estavam em tratamento e que apresentaram PA controlada, observou-se que idosos com menos de um ano de estudo apresentaram menor controle da PA (30\%) em relação aos que tinham um ou mais anos de estudo (50\%). Em estudo realizado em São José do Rio Preto, SP, Moreira et al. ${ }^{23}$ observaram taxas semelhantes em indivíduos com baixa escolaridade na faixa etária de 40-49 anos.

Apesar das evidências de que o tratamento anti-hipertensivo é eficaz em diminuir a morbidade e a mortalidade cardiovascular, os percentuais de controle da PA observados foram muito baixos. Outros estudos observaram percentuais de controle de $20 \%$ a $40 \% .^{5}$ Neste estudo, 43,5\% dos hipertensos em tratamento apresentavam PA controlada. Este achado é inferior ao observado em São José do Rio Preto, $\mathrm{SP}^{23}$ superior aos achados de Cuba e Índia e consistente com estudos realizados na China. ${ }^{21}$

Este estudo apresenta algumas vantagens e limitações. A principal vantagem foi ter sido realizado por meio de inquérito de base populacional, no qual foi possível obter informações de uma amostra representativa da população, não se limitando à demanda dos serviços de saúde. Monitorar a situação de saúde da população idosa proporciona, além do avanço do conhecimento, importante estratégia para melhorar a qualidade de saúde e de vida dos idosos, por meio da formulação e avaliação das políticas públicas de saúde para pessoas idosas. Por outro lado, considerando o delineamento deste estudo, uma das limitações foi a impossibilidade de se afirmar a temporalidade dos fatores associados encontrados. No entanto, o controle das variáveis confundidoras permitiu estabelecer algumas associações entre as variáveis estudadas e o desfecho de interesse - a hipertensão arterial.

\section{CONCLUSÃO}

Este estudo representa uma contribuição adicional para o conhecimento sobre a hipertensão arterial no Brasil, mostrando sua prevalência, fatores associados e o reconhecimento sobre a condição de hipertensão, tratamento e controle da doença em idosos. Estudos nesta área do conhecimento são importantes para o planejamento e o estabelecimento de políticas públicas em benefício da população idosa.

Apesar de, nos últimos anos, o Brasil registrar avanços por meio da implementação de políticas abrangentes para as doenças crônicas não transmissíveis (DCNT) e seus fatores de risco, geralmente estas são direcionadas à população geral, sem a necessária ênfase à população idosa. Ainda que as políticas públicas devam contemplar todos, atenção especial deve ser destinada aos grupos mais vulneráveis, tanto em ações preventivas e terapêuticas, para o controle da hipertensão arterial, quanto de promoção da saúde geral da população idosa.

\section{AGRADECIMENTOS}

À Coordenação de Aperfeiçoamento de Pessoal de Nível Superior (CAPES), pelo auxílio financeiro; ao Ministério da Saúde do Brasil e ao Conselho Nacional de Desenvolvimento Científico e Tecnológico (CNPq) - Brasil/processo $\mathrm{n}^{\circ}$ 402879/2005-8, pelo financiamento do projeto; à Secretaria de Estado de Saúde de Mato Grosso e às prefeituras dos municípios de Alta Floresta, Sinop, Sorriso e Diamantino, pelo apoio à pesquisa. 


\section{REFERÊNCIAS}

1. Organização Mundial da Saúde. Envelhecimento ativo: uma política de saúde. Brasília: Organização Pan-Americana da Saúde; 2005.

2. Schmidt MS, Duncan BB, Silva GA, Menezes AM, Monteiro CA, Barreto SM et al. Doenças crônicas não transmissíveis no Brasil: carga e desafios atuais. The Lancet 2011:61-74.

3. World Health Organization. Global health risks: mortality and burden of disease attributable to selected major risks. Geneva: WHO; 2009 [acesso em 10 mar 2010]. Disponível em: www. who.int/healthinfo/global_burden_disease/ GlobalHealthRischs_report_full.pdf.

4. Veras R. Envelhecimento populacional contemporâneo: demandas, desafios e inovações. Rev Saúde Pública 2009;43(3):548-54.

5. VI Diretrizes Brasileiras de Hipertensão Arterial. Arq Bras Cardiol 2010;95(1 supl1):1-51.

6. Gravina CF, Grespan SM, Borges JL. Tratamento não-medicamentoso da hipertensão no idoso. Rev Bras Hipertens 2007;14(1):33-6.

7. Gus I, Harzheim E, Zaslavsky C, Medina C, Gus M. Prevalência, reconhecimento e controle da hipertensão arterial sistêmica no estado do Rio Grande do Sul. Arq Bras Cardiol 2004;83(5):424-8.

8. Brasil. Ministério da Integração Nacional. Plano de desenvolvimento regional sustentável para a área de influência da rodovia BR-163: oficina de consulta à sociedade local. Brasília: Casa Civil da Presidência da República; 2004 [acesso em 20 fev 2010]. Disponível em: http://portal.saude.gov.br/portal/arquivos/pdf/ BR163DOCConsulta_a.pdf.

9. Marcopito LF, Rodríguez SSF, Pacheco MA, Shirassu MM, Goldfeder AJ, Moraes MA. Prevalência de alguns fatores de risco para doenças crônicas na cidade de São Paulo. Rev Saude Publica 2005;39(5):738-45.

10. Zaitune MPA, Barros MBA, César CLG, Carandina L, Goldbaum M. Fatores associados ao sedentarismo no lazer em idosos, Campinas, São Paulo, Brasil. Cad Saude Publica 2007;23(6):1329-38.

11. Gibson RS. Principles of nutritional assessment. New York: Oxford University Press; 1990. 712 p.

12. Callaway CW, Chumlea WC, Bouchard C, Himes JH, Lohman TG, Martin AD, et al. Circumferences. In: Lohman TG, Roche AF, Martorell R, editores. Anthropometric standardization reference manual. Champaign, Illinois: Human Kinetics Books; 1988. p. $39-54$.
13. World Health Organization. Obesity: preventing and managing the global epidemic. WHO Technical Report Series 894. Geneva: WHO; 2000 [acesso em 20 fev 2010]. Disponível em: http://www.who.int/ nutrition/publications/obesity/WHO_TRS_894/en/ index.html

14. V Diretrizes Brasileiras de Hipertensão Arterial. Arq Bras Cardiol 2007;89(3):E27-79.

15. Francisco PMSB, Donalisio MR, Barros MBA, Cesar CLG, Carandina L, Goldbaum M. Medidas de associação em estudo transversal com delineamento complexo: razão de chances e razão de prevalência. Rev Bras Epidemiol 2008;11(3):347-55.

16. Rosário TM, Scala LCN, França GVA, Pereira MR, Jardim PCBV. Fatores associados à hipertensão arterial sistêmica em Nobres - MT. Rev Bras Epidemiol 2009;12(2):248-57.

17. Nascente FMN, Jardim PCBV, Peixoto MRG, Monego ET, Moreira HG, Vitorino PVO, et al. Hipertensão arterial e sua correlação com alguns fatores de risco em cidade brasileira de pequeno porte. Arq Bras Cardiol 2010;95(4):502-9.

18. Cipullo JP, Martin JFV, Ciorlia LAS, Godoy MRP, Cação JC, Loureiro AAC, et al. Prevalência e fatores de risco para hipertensão em uma população urbana brasileira. Arq Bras Cardiol 2010;94(4):519-26.

19. Jardim PCBV, Gondim MRP, Monego ET, Moreira HG, Vitorino PVO, Souza WKSB, et al. Hipertensão arterial e alguns fatores de risco em uma capital brasileira. Arq Bras Cardiol 2007;88(4):452-7.

20. Redón J, Cea-Calvo L, Lozano JV, Martí-Canales JC, Llisterri JL, Gonzájez-Esteban J. Blood pressure and estimated risk of stroke in the elderly population of Spain: The PREV-ICTUS study. Stroke 2007;38(4):1167-73.

21. Prince MJ, Ebrahim S, Acosta D, Ferri CP, Guerra M, Huang $\mathrm{Y}$, et al. Hypertension prevalence, awareness, treatment and control among older people in Latin America, India and China: a 10/66 cross-sectional population-based survey. J Hypertens 2012;30(1):177-87.

22. Martins MSAS, Ferreira MG, Guimarães LV, Vianna LAC. Hipertensão arterial e estilo de vida em Sinop, município da Amazônia Legal. Arq Bras Cardiol 2010;94(5):639-44.

23. Moreira GC, Cipullo JP, Martin JFV, Ciorlia LAS, Godoy MRP, Cesarino CB, et al. Evaluation of the awareness, control and cost-effectiveness of hypertension treatment in a Brazilian city: populational study. J Hipertens 2009;27(9):1900-7. 
24. Barbosa AR, Borgatto AF. Arterial hypertension in the elderly of Bridgetown, Barbados: prevalence and associated factors. J Aging Health 2010;22(5):611-30.

25. Brindel P, Hanon O, Dartigues JF, Ritchie K, Lacombe JM, Ducimetière P, et al. Prevalence, awareness, treatment, and control of hypertension in the elderly: the Three City study. J Hipertens 2006;24(1):51-8.

26. Dupre ME, Beck AN, Meadows SO. Marital trajectories and mortality among US adults. Am J Epidemiol 2009;170(5):546-55.

27. Mastroeni MF, Mastroeni SSBS, Erzinger GS, Marucci MFN. Antropometria de idosos residentes no município de Joinville-SC, Brasil. Rev Bras Geriatr Gerontol 2010;13(1):29-40.

28. Silveira EA, Kac G, Barbosa LS. Prevalência e fatores associados à obesidade em idosos residentes em Pelotas, Rio Grande do Sul, Brasil: classificação da obesidade segundo dois pontos de corte do índice de massa corporal. Cad Saude Publica 2009;25(7):1569-77.

29. Costa MFFL, Peixoto SV, César CC, Malta DC, Moura EC. Comportamentos em saúde entre idosos hipertensos, Brasil, 2006. Rev Saude Publica 2009;43(supl 2):18-26.
30. Munaretti DB, Barbosa AR, Marucci MFN, Lebrão ML. Hipertensão arterial referida e indicadores antropométricos de gordura em idosos. Rev Assoc Med Bras 2011;57(1):25-30.

31. Pimenta AM, Kac G, Gazzinelli A, Corrêa-Oliveira R, Velásquez-Meléndez G. Associação entre obesidade central, triglicérides e hipertensão arterial em uma área rural do Brasil. Arq Bras Cardiol 2008;90(6):419-25.

32. Oliveira SMJV, Santos JLF, Lebrão ML, Duarte YAO, Pierin AMG. Hipertensão arterial referida em mulheres idosas: prevalência e fatores associados. Texto Contexto Enferm 2008;17(2):241-9.

33. Coylewright M, Reckelhoff JF, Ouyang P. Menopause and hypertension: an age-old debate. Hypertension 2008;51:952-9.

34. Banegas JR, Guallar-Castillón P, Rodríguez-Artalejo F, Graciani A, López-García E, Ruilope LM.

Association between awareness, treatment, and control of hypertension, and quality of life among older adults in Spain. Am J Hipertens 2006;19(7):686-93.

35. Ong KL, Cheung BM, Man YB, Lau CP, Lam KS. Prevalence, awareness, treatment, and control of hypertension among United States adults 1999-2004. Hypertension 2007;49(1):69-75. 